\title{
Geografías de la diversidad sexogenérica más allá de la gran ciudad: experiencias, discursos y prácticas en dos ciudades medianas de Cataluña
}

\author{
Jose Antonio Langarita Adiego \\ Universitat de Girona \\ josan.langarita@udg.edu \\ Jordi Mas Grau \\ Olga Jubany \\ Universitat de Barcelona \\ jordimasgrau@ub.edu \\ lga.jubany@ub.edu
}

Recibido: noviembre de 2018

Aceptado: abril de 2018

Publicado: junio de 2019

\section{Resumen}

La gran ciudad se ha presentado mediáticamente, y también en las líneas predominantes del debate académico, como el espacio propio para las personas LGTB. Se concibe como el único lugar vivible y de experimentación para este grupo poblacional, absorbiendo así los significados de la diversidad sexogenérica. En este artículo se analizan las experiencias, los discursos, las representaciones y las prácticas de personas LGTB que viven fuera de la gran ciudad (Barcelona), a través del estudio de dos poblaciones medianas (Girona y Sabadell), con el fin de abordar dos asuntos clave: (1) los vínculos, las relaciones y las influencias entre ciudades medianas y la gran ciudad en materia de diversidad sexual y de género, y (2) la construcción de alteridades socioculturales en las percepciones sobre la tolerancia hacia las expresiones LGTB a través de la relación entre el centro y las periferias urbanas. Dicho análisis se fundamenta en los resultados empíricos de una investigación de corte cualitativo basada en entrevistas en profundidad, grupos de discusión y observación participante.

Palabras clave: espacio urbano; estudios LGTB; ciudades medianas; LGTBfobia; etnografía 
Resum. Geografies de la diversitat sexogenèrica més enllà de la gran ciutat: experiències, discursos i pràctiques en dues ciutats mitjanes de Catalunya

La gran ciutat s'ha presentat mediàticament, i també en les línies predominants del debat acadèmic, com l'espai propi per a les persones LGTB. Es concep com l'únic lloc habitable i d'experimentació per a aquest grup poblacional, amb la qual cosa acapara els significats de la diversitat sexogenèrica. En aquest article s'hi analitzen les experiències, els discursos, les representacions i les pràctiques de persones LGTB que viuen fora de la gran ciutat (Barcelona), a través de l'estudi de dues poblacions mitjanes (Girona i Sabadell), a fi d'abordar dos assumptes clau: (1) els vincles, les relacions i les influències entre ciutats mitjanes i la gran ciutat en matèria de diversitat sexual i de gènere, i (2) la construcció d'alteritats socioculturals en les percepcions sobre la tolerància vers les expressions LGTB a través de la relació entre el centre i les periferies urbanes. Aquesta anàlisi es fonamenta en els resultats empírics de recerca de caire qualitatiu basada en entrevistes en profunditat, grups de discussió i observació participant.

Paraules clau: espai urbà; estudis LGTB; ciutats mitjanes; LGTBfobia; etnografia

Résumé. Géographies de la diversité sexuelle et de genre en dehors des grandes villes: Expériences, discours et pratiques dans deux villes moyennes de la Catalogne

Les grandes villes sont présentées dans les médias, et dans certaines études universitaires également, comme l'espace de référence des personnes LGBT. Elles apparaissent comme le seul lieu vivable et d'expérimentation pour ce groupe de population, s'appropriant de ce fait les représentations de la diversité sexuelle et de genre. Dans cet article, à travers l'étude de deux villes de taille moyenne (Girone et Sabadell), nous analysons les expériences, discours, représentations et pratiques des personnes LGBT vivant en dehors de la métropole (Barcelone) afin d'aborder deux sujets clés : (1) les liens, relations et influences entre les villes de taille moyenne et les grandes villes en termes de diversité sexuelle et de genre, et (2) la construction d'altérations socioculturelles dans la perception de la tolérance envers les expressions LGBT, à travers la relation entre le centre et les périphéries urbaines. Dans cet objectif, une recherche qualitative basée sur des entretiens approfondis, des groupes de discussion et des observations de participants a été réalisée.

Mots-clés: espace urbain; études LGBT; villes moyennes; LGBTphobie; ethnographie

Abstract. Geographies of sexual and gender diversity beyond the big city: Experiences, discourses and practices in two mid-sized cities of Catalonia

The big city has been presented in the media and some academic research as the ideal space for LGBT people. Indeed, the big city is conceived as the only livable and experimental place for sexual and gender diversity, thus monopolizing the meanings of gender-generic diversity. Grounded in a study of two mid-sized cities (Girona and Sabadell) that focused on the experiences, discourses, representations and practices of LGTB people living outside the big city (Barcelona), and using qualitative data gathered in-depth interviews, discussion groups and participant observation, this paper analyzes and discusses two fundamental issues. Firstly, the links, relationships and influences between mid-sized cities and the big city in terms of sexual and gender diversity; and secondly, the construction of alterities through the relationship between the centers and urban peripheries of these two cities.

Keywords: urban space; LGBT studies; mid-sized cities; LGBTphobia; ethnography 


\section{Sumario}

1. Introducción

2. Consideraciones metodológicas

3. Notas de contexto

4. A la sombra de la gran ciudad: vidas LGTB fuera de Barcelona
5. El homófobo es el otro: desigualdades socioespaciales en la percepción de la LGTBfobia

6. Conclusiones: pensando desde fuera de la gran ciudad

Referencias bibliográficas

\section{Introducción}

La distinción entre espacio público y espacio privado para las personas lesbianas, gais, trans y bisexuales (LGTB) no está tan marcada como para otros grupos sociales, ya que los mecanismos y los ejercicios de control sobre la normatividad sexogenérica se producen tanto en el espacio público como en el privado (Eribon, 2001). De hecho, el espacio privado ha sido históricamente uno de los escenarios de mayor opresión para muchas personas LGTB. Son innumerables las historias vinculadas con las «salidas del armario» de personas LGTB que refieren a graves problemas y violencias en los entornos de proximidad. Sin embargo, el espacio público también es especialmente significativo para estas personas, en la medida en que es el lugar de visibilización, socialización y construcción de referencias colectivas. En este sentido, la ciudad — en especial, la gran urbe- ha sido el lugar imaginado como el espacio para la liberación de las personas LGTB, hasta el punto de establecerse una conexión casi inseparable entre la diversidad sexogenérica y las grandes ciudades occidentales. Con todo, esta representación resulta problemática, puesto que además de ser profundamente etnocéntrica, invisibiliza otras realidades LGTB que no se articulan en estos contextos urbanos.

En la década de 1970 se publica el primer trabajo sobre diversidad sexual y espacio urbano de la mano de Bonnie Loyd y Lester Rowntree (1978). Sin embargo, es el libro de Manuel Castells (1983) el considerado como el texto fundacional de los análisis sobre las cuestiones sexuales en los estudios urbanos. A raíz de ello, se va generando un debate que da sus frutos a partir de los años 90, con diferentes investigaciones que centran su atención en las cuestiones sexogenéricas en tanto que dimensiones del poder, la identidad y la desigualdad que determinan los fenómenos socioespaciales de las ciudades. Es gracias a las contribuciones de Adler y Brenner (1992), Baley (1999), Bell y Valentine (1995), Chauncey (1994), Higgs (1999), Hubbard (1999), Ingram et al. (1997), Knopp (1992, 1994, 1998), Mort y Nead (1999), Probyn (1995), Weston (1995) y Whittle (1994), entre otros, que la relación entre espacios urbanos, género y sexualidad se consolida como un campo de investigación propio de las ciencias sociales. A partir de comienzos del siglo XXI se multiplican las investigaciones sobre sexualidad y espacio urbano, lo que produce importantes resultados científicos en disciplinas tales como la antropología, la sociología, la geografía o la economía.

Las grandes ciudades occidentales han sido el principal objeto de análisis de los estudios urbanos sobre sexualidad (ver Aldrich, 2004; Black et al., 2002; 
Hubbard, 1999; Nash, 2013; Sibalis, 2004; Hubbard y Wilkinson, 2015, entre otros). Aunque existen algunas investigaciones que han abordado la cuestión fuera de las grandes metrópolis (ver Bell, 2000; Browne, 2011; Kazyak, 2011; Kuhar y Švab, 2014; Mcglynn, 2018; Muller, 2013; Phillips et al., 2000; Wienke y Hill, 2013), las teorías, los discursos y las representaciones sobre la diversidad sexual y de género se han centrado en la gran ciudad, dando por descontado que es el lugar desde el cual, supuestamente, se construye la identidad y el estilo de vida propio de las personas LGTB (ver Bech, 1997; Chauncey, 1994; Hindle, 1994; Weston, 1995).

Las grandes ciudades monopolizan las representaciones y los discursos sobre «lo LGTB», determinan la agenda política del movimiento de liberación, atraen el interés mediático y concentran los grandes eventos y los espacios de socialización específicos como bares, restaurantes o discotecas. Al fin y al cabo, la gran ciudad se presenta como el lugar «natural y deseable» para estas personas. En consecuencia, se ha consolidado la idea de que las personas LGTB que no viven en una gran ciudad están en desventaja con respecto a las que residen en la gran urbe. Se considera una elección vital desfavorable el hecho de residir en territorios de menor tamaño, a los que se atribuye una mayor hostilidad hacia la diversidad sexogenérica (Wienke y Hill, 2013).

Sin embargo, esta centralidad no ha estado exenta de críticas. Halberstam (2005) problematiza la gran ciudad como espacio de producción de privilegios y de normatividades, lo que ha llamado «metronormatividad». Por su parte, Gavin Brown (2008 y 2012) también cuestiona la hegemonía de la gran ciudad y critica la aplicabilidad de la noción de homonormatividad de Lisa Duggan (2002) para entender las experiencias cotidianas de muchas personas LGTB que tienen vidas «ordinarias». Asimismo, hay quienes critican la dicotomía simplificadora que establece el espacio urbano como "LGTBfriendly» y el rural como LGTBfóbico ${ }^{1}$ (Champion y Hugo, 2016).

Al fin y al cabo, no todas las personas LGTB viven en grandes ciudades, y las que no lo hacen no están inexorablemente condenadas al aislamiento, el rechazo o la abstinencia sexual. Si aceptamos que las vidas LGTB solo son vivibles en las grandes ciudades, estamos negando la existencia de otras experiencias que no están atravesadas por la gran urbe. Los mensajes apocalípticos acerca de las personas LGTB que viven fuera de la gran ciudad están cargados de prejuicios que hay que discutir y someter a un análisis más profundo. Es necesario entender la complejidad de la diversidad sexual y de género no solo en lo referente a la cuestión identitaria o en su intersección con otras opresiones, sino también desde la dimensión territorial y geográfica en la que se enmarca.

1. A lo largo del texto utilizamos la palabra LGTBfobia para referirnos al «conjunto de creencias culturales y personales, opiniones, actitudes y comportamientos agresivos basados en los prejuicios, repulsión, miedo y/u odio dirigida contra personas o grupos que no corresponden a las normas y expectativas sociales de género y sexualidad o que la transgreden» (Herramientas para personas LGTB que residen en ciudades pequeñas y medianas, s. f. Recuperado el 25 de abril de 2019 en la página web: <http://divercity.ub.edu/publications/>). 
Por otro lado, resulta importante destacar que esta discusión teórica se ha producido fundamentalmente en el marco académico anglosajón. En el contexto español, los estudios urbanos con datos empíricos sobre el territorio todavía son muy incipientes (ver Coll-Planas, 2017; Rodó-de-Zárate, 2015). Es por ello por lo que siguen siendo necesarias las aproximaciones que permitan visibilizar y analizar dicha diversidad en otras geografías y contextos socioculturales. Asimismo, si aceptamos que se ha producido una colonización discursiva y de representación LGTB por parte de la gran ciudad, es inevitable admitir las influencias —en las prácticas y subjetividades - de las dinámicas globales a través de las relaciones internacionales (Boellstorff y Leap, 2004). No obstante, ello no significa la existencia una «sociedad LGTB occidental», con los mismos intereses, preocupaciones y estilos de vida. Más bien debemos entender que buena parte de estas subjetividades, prácticas y representaciones globalizadas se resignifican en los procesos de socialización LGTB de los contextos locales (Cáceres Feria y Valcuende del Río, 2014; Vasquez del Aguila, 2014). Pensar en una comunidad LGTB global sin considerar las particularidades es no dar cuenta de las diferencias de clase, identidades racializadas, edad, capacidades, y también territoriales, que envuelven las experiencias de las personas LGTB (ver Platero, 2012).

Este artículo analiza cómo se construyen las representaciones, los discursos y las prácticas de las personas LGTB residentes en dos ciudades medianas de Cataluña: Sabadell y Girona. Si bien este análisis tiene por objeto repensar la hegemonía de la gran ciudad en las vidas de las personas LGTB que no residen en ella, no se pueden ignorar las interacciones ni las tensiones existentes entre la gran ciudad de referencia de la región (Barcelona) y las poblaciones de menor tamaño. Un segundo objetivo consiste en analizar las percepciones que tienen las personas LGTB respecto a la tolerancia —o la hostilidad — hacia la diversidad sexogenérica predominante en determinadas zonas de ambas ciudades. Como se verá, estas percepciones socioespaciales configuran en el imaginario colectivo la idea de zonas seguras e inseguras en base a la producción de alteridades culturales y económicas. Con todo, hay que tener en cuenta que las trayectorias personales, el capital cultural y las redes sociales de cada sujeto influyen notablemente en la construcción de esa alteridad «homófoba», por lo que deben considerarse a la hora de realizar cualquier análisis que pretenda ser riguroso.

\section{Consideraciones metodológicas}

Los datos para la elaboración de este artículo proceden de una investigación etnográfica llevada a cabo en el marco del proyecto de investigación Divercity: Prevenir y combatir la homo- y la transfobia en pequeñas y medianas ciudades de Europa (2015-2017)2. Además de Sabadell y Girona, participaron en el proyecto las ciudades de Charleroi (Bélgica), Tesalónica (Grecia), Nottingham

2. Proyecto cofinanciado por el programa de Derechos, Igualdad y Ciudadanía de la Unión Europea (JUST/2014/RRAC/AG/6693). Para más información sobre el proyecto, consúltese su página web (<http://divercity.ub.edu/>). 
(Reino Unido) y Breslavia (Polonia). La fase analítica tenía por objeto analizar las experiencias y los discursos vinculados a la LGTBfobia en dichas ciudades, así como las medidas de índole política, legal y social puestas en marcha para combatir este problema social. Las técnicas de investigación empleadas fueron las entrevistas en profundidad, los grupos de discusión y la observación participante.

En el caso de Girona y Sabadell se realizaron un total de 58 entrevistas (34 a personas LGTB y 24 a cuadros políticos, representantes de organizaciones LGTB y profesionales cuyas responsabilidades inciden directamente en el bienestar de este sector poblacional). Asimismo, se organizaron cuatro grupos de discusión (dos con personas LGTB y dos más con políticos, profesionales y representantes de colectivos). Finalmente, se llevaron a cabo diversas sesiones de observación participante durante las movilizaciones por días señalados (como el 28 de junio o las concentraciones de repulsa al atentado en la discoteca norteamericana Pulse ${ }^{3}$ ), en espacios de ocio y socialización LGTB y en actividades organizadas por los gobiernos locales, como la Mesa de Género, Feminismos y LGTBI de Sabadell o el Consell Municipal LGTBI de Girona.

El acceso a las personas participantes se llevó a cabo a partir de contactos personales, contactos facilitados por las administraciones públicas y las entidades sociales, relaciones establecidas durante las sesiones de observación y/o a través de las aplicaciones en línea. Dichas estrategias fueron combinadas con la técnica de la bola de nieve, lo que permitió ampliar y diversificar la muestra.

\section{Notas de contexto}

Girona es una ciudad de 99.000 habitantes situada en el noroeste de la Comunidad Autónoma de Cataluña, a unos $100 \mathrm{~km}$ por carretera de Barcelona, capital de la provincia homónima y centro del Área Urbana de Girona. La renta per cápita de la ciudad (28.837 euros) es superior a la media de Catalunya $(27.540 \text { euros })^{4}$, mientras que la tasa de paro registrado (un 9,17\%) se sitúa por debajo de la media de la Comunidad (un 11,4\%) . El asociacionismo LGTB, como el del resto de Cataluña a excepción de Barcelona, está muy debilitado y con dificultades para interpelar al conjunto de la sociedad. Cabe destacar que, recientemente, desde el Ayuntamiento de la ciudad se han desplegado algunas medidas dirigidas a la población LGTB, como la creación del Consell Municipal LGTBI ${ }^{6}$, la conmemoración de algunos días señalados o la cesión de un

3. Este espacio LGTB-friendly de Orlando sufrió un atentado homófobo el 12 de junio de 2016, en el que murieron 50 personas y 53 resultaron heridas.

4. Fuente: El Pais (<https://elpais.com/especiales/2016/renta-per-capita-municipios-espana/\#278>).

5. Fuente: Encuesta de Población Activa (EPA).

6. A pesar de que a lo largo de texto utilizamos el acrónimo LGTB, en el caso del Consell Municipal utilizamos la expresión LGTBI con el fin de respetar el nombre original de dicho organismo. 
local a las entidades LGTB. Además, se ha puesto en marcha la activación de un servicio de atención integral (SAI) dirigido a las personas LGTB.

Por su parte, Sabadell es una ciudad de 209.000 habitantes perteneciente al Área Metropolitana de Barcelona (está situada a escasos $25 \mathrm{~km}$ de la capital). Su renta per cápita (25.837 euros) es inferior a la media catalana, mientras que su tasa de paro se sitúa prácticamente a la par (un 11,3\%). Si bien ya hace algunos años que se organizan actos en favor de las expresiones LGTB, como el izado de la bandera del arcoíris en las fechas señaladas o el día contra la homofobia en el fútbol, las políticas de carácter estructural para potenciar la diversidad sexual y de género (como el Plan Estratégico) constituyen una esfera de gobierno reciente. Por tanto, y al igual que sucede con Girona, todavía es demasiado pronto para efectuar una evaluación exhaustiva de dichas políticas.

\section{A la sombra de la gran ciudad: vidas LGTB fuera de Barcelona}

Barcelona concentra el 21\% de la población de la región (porcentaje que se eleva hasta el $43 \%$ si tomamos como referencia su área metropolitana). La Ciudad Condal es sede de las grandes firmas empresariales y financieras de Cataluña y aglutina los principales órganos del gobierno autonómico, la mayoría de organizaciones de la sociedad civil y gran parte de los centros de producción cultural de la región. Todo lo que acontece en Barcelona importa y acapara de inmediato el interés mediático de toda Cataluña.

En lo referente al universo LGTB, la centralidad de Barcelona también resulta significativa. Dicha ciudad desarrolla un papel crucial en la lucha por los derechos de las minorías sexuales y de género de toda España. En ella se produce el primer intento clandestino de organización homosexual durante la dictadura franquista (1970) y poco más tarde se fundan las primeras asociaciones de personas homosexuales (1975) y trans (1978) de España. De ahí que en las calles barcelonesas tiene lugar la primera manifestación por la liberación homosexual del país, en 1977 (Mira, 2004; Ortega y Platero, 2015).

Asimismo, desde inicios del siglo Xx la ciudad de Barcelona es lugar de encuentro para todo tipo de disidentes sexogenéricos. El Barrio Chino (hoy renombrado como el Raval) y la avenida del Paral.lel desprenden un aire canalla que atrae a personajes ávidos de experiencias, como Jean Genet o Guy Hocquenghem. Sus callejuelas, bares y teatros se convierten en espacios furtivos de libertad en tiempos de la dictadura franquista, caracterizados por una fuerte represión política, policial y judicial (Huard, 2014; Mérida Jiménez, 2016; Mira, 2004). Recientemente, el llamado Gaixample de Barcelona es una de las principales zonas de ocio gay de Europa, en donde se ofrecen todo tipo de bienes y servicios fundamentalmente para gais con cierto poder adquisitivo, aunque también existen algunos espacios y servicios que acostumbran a utilizar lesbianas y personas trans ${ }^{7}$.

7. Gaixample es el término resultante de unir los vocablos gai y Eixample, que es el nombre del distrito en donde se ubica esta zona de ocio. 
El hecho de que Barcelona sea un referente histórico de la lucha LGTB en España y que concentre en la actualidad la mayoría de servicios y organizaciones LGTB de Cataluña, ejerce un marcado influjo, tanto en el imaginario colectivo como en las vidas de las personas LGTB. Por un lado, los proyectos y las iniciativas LGTB que tienen lugar en Barcelona monopolizan la atención pública, mientras que aquellas experiencias fuera de la capital son a menudo ignoradas por los medios y los discursos capitalinos, principales productores de relatos y significados. Por otro lado, y como consecuencia de lo anterior, personas procedentes de otras zonas de Cataluña, y también del resto de España, se «sexilian» ${ }^{8}$ en Barcelona con la ilusión de desarrollar más libremente su orientación sexual y/o su identidad de género y encontrar espacios de afinidad, ocio y activismo político.

La migración a la gran ciudad por parte de las personas LGTB no es un fenómeno nuevo y diversos estudios han puesto su atención en este hecho (ver Annes y Redlin, 2012; Fountain-Stokes, 2004; Hibbins, 2005; Langarita Adiego y Salguero Velázquez, 2016; Smith, 2012, entre otros). Sin embargo, es necesario destacar que, a pesar de la relevancia del sexilio en los procesos de construcción de la identidad colectiva, así como en la construcción de las narrativas LGTB, no todas las personas LGTB ven en la migración a la gran ciudad la vía más adecuada para mejorar su bienestar o encontrar espacios de socialización. La migración hacia las grandes ciudades no es un proyecto vital necesario para las personas LGTB, ya que muchas de ellas no proyectan sus existencias en los grandes entornos urbanos. Por tanto, aunque se trate de un fenómeno conocido y significativo para muchas personas LGTB, no es una realidad extendida al conjunto de la población LGTB.

Para entender los casos de Girona y Sabadell debemos considerar que a la proximidad geográfica de Barcelona (100 km y $25 \mathrm{~km}$, respectivamente) se le ha de añadir una importante dependencia sociocultural de la capital. Como resultado, en ambas ciudades existe una débil presencia de organizaciones y espacios LGTB. Este rasgo es más significativo en el caso de Sabadell, debido en buena medida a su mayor cercanía y a las excelentes conexiones viales y ferroviarias con la capital (en 30 minutos se está en el centro de Barcelona). Si bien el tren de alta velocidad también conecta Girona y Barcelona en poco más de media hora, el elevado precio del billete impide a muchas personas ir y volver en un mismo día con asiduidad?

En el momento de realizar el trabajo de campo (febrero-noviembre de 2016) no existe en Sabadell ningún espacio de ocio o de socialización ni ninguna organización estrictamente $\mathrm{LGTB}^{10}$. Con todo, para parte de las personas

8. El término sexilio fue acuñado por Manolo Guzmán (1997) y posteriormente Yolanda Martínez-San Miguel (2011) analizó los alcances semánticos del concepto.

9. Con el tren convencional se tarda entre una hora y veinte y una hora y cuarenta minutos para ir de Girona a Barcelona.

10. Sin embargo, cuando se redacta este artículo se ha tenido constancia de la reciente creación de Ca l'Enredus, un movimiento asociativo LGTBIQ+ que tiene por objetivo ofrecer un espacio de acompañamiento integral y constituirse en una red local e intercomunitaria para 
entrevistadas, esta ausencia no es motivo de preocupación, ya que prefieren aprovechar los recursos, los espacios y los servicios de Barcelona. Según su experiencia, la capital les aporta un anonimato que no encuentran en su ciudad: «Sabadell, sobre todo el centro, es como un pueblo y todo el mundo se conoce» (hombre gay, 24 años). El anonimato de la gran ciudad confiere una sensación de libertad y una desinhibición que facilitan las muestras públicas de afecto, reprimidas a menudo en los espacios de proximidad por el exceso de control social: "Hay gente gay de Sabadell que se va a Barcelona para "darse el lote" nada más bajarse del tren» (mujer trans bisexual, 30 años). Sucede algo similar con las zonas de ocio y socialización, en las que se valora no solo el anonimato, sino también la diversidad: «La gente va a Barcelona. Cuando alguien va a un bar quiere conocer a gente nueva y ser anónimo. Un bar en Sabadell no te permite conocer a gente nueva, ni ser anónimo» (hombre gay, 38 años).

En lo relativo a la ausencia de asociaciones LGTB, las personas entrevistadas de Sabadell añaden dos motivos al ya mencionado influjo de Barcelona. Se recurre en primer lugar a un argumento que se ha generalizado en los últimos tiempos en muchos países de la Europa occidental. Se trata de la extensión de un sentimiento de conformismo y completitud entre parte de la población LGTB tras lograr derechos como los del matrimonio y la adopción, lo que ha generado un efecto desmovilizador que afecta a la actividad de las organizaciones (Mas Grau y Jubany, 2019): «Muchos homosexuales ya no necesitan pertenecer a un grupo para reivindicar derechos. Hacen su vida de pareja, quieren vivir su sexualidad con normalidad» (hombre gay, 64 años). La segunda razón que explicaría la falta de asociaciones LGTB está relacionada con la fuerte presencia de movimientos sociales y asociativos en la ciudad. De este modo, algunas personas destacan que en Sabadell hay muchas organizaciones que promueven valores como la igualdad, la solidaridad y la lucha contra la discriminación, con lo que constituyen espacios de seguridad para la expresión y la reivindicación de la diversidad sexogenérica:

Me da la impresión de que es una ciudad en la que hay bastante compromiso y mucha gente organizada, ya sean grupos anarquistas, anticapitalistas o feministas. Es posible que la comunidad LGTB esté disuelta en todos esos grupos, que ya son lo suficientemente respetuosos con el tema de la diversidad sexual para que no haga falta que haya una entidad propia. (Profesional de una ONG de Sabadell)

Si bien muchas de las personas entrevistadas no lamentan la falta de espacios de socialización específicos para la población LGTB de Sabadell, para otras sí que sería importante que el Ayuntamiento o la sociedad civil crearan algún

personas y colectivos sensibilizados con la diversidad sexogenérica. Ca l'Enredus ha recibido el apoyo económico del Ayuntamiento. Es necesario señalar que, al igual que en el caso del Consejo Municipal LGBTI del Ayuntamiento de Girona, nos referimos a Ca l'Enredus como organización LGTBIQ+ para respetar el término que ellos emplean a través de las redes sociales. 
espacio en el que poder relacionarse y encontrar apoyo y asesoramiento. Esta necesidad se detecta especialmente en aquellas personas que se encuentran en las primeras etapas de su desarrollo sexogenérico y que no cuentan con un entorno afectivo favorable:

Me habría gustado que Sabadell tuviera espacios para conocer a otras lesbianas en la época en que salí del armario. En ese momento estaba perdida: no hablaba con mi familia, no conocía a nadie y no sabía adónde ir. (Mujer lesbiana, 41 años)

Al igual que Sabadell, Girona se encuentra bajo la influencia de la gran ciudad de Barcelona, aunque la mayor distancia y su condición de capital de provincia le confieren algunas particularidades que la diferencian de Sabadell. Del mismo modo que los habitantes de esta última, muchos gerundenses LGTB viajan a Barcelona para disfrutar de su oferta de ocio. Sin embargo, las mayores dificultades para desplazarse a diario a la capital, junto con una mayor sensación de control social al tratarse de una ciudad más pequeña, hacen que la necesidad de trasladarse a vivir a Barcelona esté más presente que en Sabadell: «Los jóvenes LGTB huyen de Girona y en cuanto pueden se van a vivir a Barcelona» (miembro de una asociación LGTB). A diferencia de Sabadell, en Girona existen diferentes asociaciones LGTB, así como feministas y transfeministas, que incorporan las reivindicaciones LGTB y queer en sus agendas políticas. Sin embargo, la participación acostumbra a ser muy baja.

Cuando se realiza el trabajo de campo existe en Girona un bar «LGBTfriendly» (actualmente cerrado). En este sentido, algunos de los relatos de las personas entrevistadas dan cuenta de la necesidad de este tipo de lugares para la emancipación y la socialización de las personas LGTB de la ciudad: «Ligar en Girona es difícil, pero es difícil porque no hay locales de ambiente» (hombre gay, 40 años). En esta cita se atribuye a los locales una función socializadora necesaria para «ligar». No obstante, desde hace algunos años, el acceso a las relaciones sexuales entre las personas LGBT, especialmente entre los hombres gais, se está desplazando a los espacios en línea y a los programas de geolocalización, con lo que se abandona la importancia de los bares y las discotecas de ambiente propios de otras épocas.

Si bien para algunas de las personas LGTB entrevistadas el sexilio hacia Barcelona ha significado vivir su sexualidad con mayor libertad y una oportunidad para alejarse del control de proximidad, para otras personas esta migración no es posible por motivos económicos. Es el caso de una mujer trans de 41 años, nacida en un pueblo de la costa de Girona, que al final decide irse a vivir a su capital de provincia debido al elevado coste de la vida barcelonesa:

Había pensado irme a vivir a Barcelona. Lo que pasa es que, en Barcelona, todos los trabajos que encontré tenían un sueldo de 800 o 900 euros. En Barcelona con 900 euros no pagas ni un piso, por eso cuando me salió el trabajo aquí en Girona, me vine. 
Con estas palabras se observa que las cuestiones económicas influyen significativamente en los procesos migratorios, incluidos los de las personas LGTB. Asimismo, se constata que las ciudades medianas no son solo el punto de origen desde el que las personas LGTB emigran a la gran ciudad, sino que, al mismo tiempo, también son territorios que reciben sexiliados de otras poblaciones de menor tamaño. De este modo, Girona "exporta» sexiliados hacia Barcelona, al tiempo que recibe una inmigración LGTB procedente de las poblaciones de su provincia. La siguiente cita refleja que el flujo migratorio LGTB no solo se dirige hacia Barcelona:

En Figueres [pequeña ciudad de la provincia de Girona] hay mucha gente de derechas. Siempre ha sido de derechas. Es horrible. Sin embargo, aquí en Girona tienes más libertad, es más grande, claro. Pero si te vas a Barcelona, que es inmenso, nadie se fija en nadie. (Mujer lesbiana, 22 años)

Pero, además, las ciudades medianas como Girona y Sabadell presentan unas ventajas singulares que las hacen apetecibles para no pocas personas LGTB, que encuentran en ellas unos lugares sin los precios ni las aglomeraciones de las grandes ciudades, pero con más servicios y equipamientos que los pequeños municipios. Es decir, las personas LGTB escogen su lugar de residencia teniendo en cuenta también otros factores que los exclusivamente relacionados con su orientación sexual y/o su identidad de género. Ello demuestra, tal y como se sostiene desde la perspectiva interseccional (ver Viveros Vigoya, 2016), que las vidas de las personas están atravesadas por ejes superpuestos de recursos, preferencias, identificaciones, limitaciones y, por supuesto, opresiones:

A mí me gusta vivir en Girona porque es grande pero no es Barcelona. Puedes encontrar de todo y es muy verde, no son todo edificios. Vivimos en una zona con un bosquecito al lado. No parece que estemos en la ciudad: ni coches ni ruido por las noches. Es muy tranquilo. ¡Girona me enamora! (Mujer lesbiana, 24 años)

Como se acaba de observar, abordar las experiencias, los discursos y las prácticas de las personas LGTB residentes en Girona y Sabadell nos lleva inevitablemente a pensarlas en su interacción con Barcelona. $\mathrm{Al}$ respecto, existe un sinfín de flujos sociales y culturales cargados de valores, expectativas, deseos y representaciones que vinculan la capital con ciudades como Girona y Sabadell que no se pueden obviar, pues contribuyen a la configuración de «lo LGTB» en las ciudades estudiadas. Así, por ejemplo, Barcelona es un punto de referencia para los gerundenses que van a barcelonear, término empleado para aquellos que van a la capital para pasear, divertirse, relacionarse y conocer las últimas novedades. Lo que, al fin y al cabo, conlleva incorporar nuevos imaginarios, perspectivas y miradas que, en el caso de las personas LGTB, todavía se sobredimensionan más por la relevancia internacional que ha tomado la ciudad de Barcelona en cuanto a servicios y espacios de socialización. Sin embargo, las influencias entre las ciudades pequeñas y las grandes ciudades no son unidirec- 
cionales, ya que buena parte de los residentes LGTB de Barcelona proceden de otros territorios, lo que también provoca ciertas influencias en la capital. Pensar que las personas LGTB abandonan sus vivencias previas cuando se trasladan a una gran ciudad es problemático, ya que los procesos de aculturación y de socialización siempre se encuentran en negociación con los valores, las experiencias y las miradas de cada persona.

\section{El homófobo es el otro: desigualdades socioespaciales en la percepción de la LGTBfobia}

En términos generales, la mayoría de las personas entrevistadas consideran que Girona y Sabadell no son especialmente hostiles hacia las expresiones LGTB. No obstante, residentes de ambas poblaciones establecen diferencias entre el centro de sus ciudades y algunos barrios periféricos cuando son preguntados por sus experiencias y sus percepciones espaciales de la LGTBfobia. Si en el imaginario colectivo se vincula a la gran ciudad con el respeto a la diversidad y al pequeño municipio con la intolerancia, entre el centro y los barrios periféricos de las ciudades se establece una distinción dicotómica similar: «Hay gente que, para "salir del armario", se va al centro de Sabadell para pasear e ir cogidos de la mano" (hombre gay, 32 años. Sabadell).

Como en tantas otras ciudades, Girona y Sabadell presentan importantes diferencias socioeconómicas entre un centro dominado por una clase media catalana de tradición burguesa y menestral, y algunos barrios de la periferia más heterogéneos — pues acogen las distintas olas migratorias - que presentan mayores índices de pobreza y paro. Asimismo, en el centro de ambas ciudades están la mayoría de comercios y zonas de restauración, por lo que concentran las actividades de ocio de muchos de sus habitantes. Las marcadas diferencias socioeconómicas entre el centro y determinados barrios periféricos acaban por adquirir una dimensión de carácter simbólico: «La gente de un barrio de la periferia no dice "voy al centro de la ciudad"; dice "voy a Sabadell” (profesional del sector educativo, Sabadell).

Algunos de los discursos identificados a lo largo de nuestra investigación para explicar la dicotomía entre el centro y la periferia presuponen un Nosotros tolerante respecto a la diversidad sexogenérica (en este caso, catalanes del centro de la ciudad), frente a un Otro embebido por una cultura esencialmente LGTBfóbica (en especial, el musulmán y el gitano de la periferia). Tal y como apunta Puar (2007), si la heteronormatividad requiere de una alteridad homosexual, la homonormatividad se contrapone a un Otro homófobo, determinado en este caso por marcadores étnico-espaciales: «En las afueras, la mayoría de gente son moros y gitanos, y ellos no aceptan las relaciones homosexuales. En cambio, en el centro de Sabadell la gente (homosexual) se puede besar e ir de la mano» (mujer lesbiana, 41 años. Sabadell). De este modo, la LGTBfobia es culturalizada, concebida como un problema consustancial de una otredad cultural a la que se teme porque que no ha interiorizado los valores - occidentales- del respeto y la tolerancia: 
Cuando has de hacer los papeles y pides el expediente de matrimonio tienes que hacer la misma cola que los de inmigración. Los juzgados abren a la misma hora y hacemos cola todos juntos [...]. Y estaba haciendo cola entre mucha gente catalana, africana, de países árabes, y pensaba: «Si supieran que yo me vengo a casar con una mujer...». ¡Sentí un miedo! (Mujer bisexual, 55 años. Girona)

Estas representaciones de un Otro LGTBfóbico incluyen a muchas de las poblaciones de origen que han protagonizado las distintas olas migratorias hacia Cataluña. Si antes se mencionaba a "africanos» y «árabes», protagonistas de las migraciones del siglo XXI, a continuación se homogeneiza a las poblaciones, mayoritariamente de origen rural, que llegan a Cataluña a partir de la década de 1960:

En el barrio donde vivo casi todos son andaluces, son extremeños, gente pobre que vino para Cataluña [...]. Esto sí que influye en la homofobia, porque la gente andaluza, eso es un tópico, yo lo sé, estoy hablando muy tópico pero sí que es verdad que son cerrados respecto a eso [la diversidad sexogenérica]. Tú vas para Andalucía y cuesta más ver según qué cosas. (Mujer bisexual, 31 años. Sabadell)

La conceptualización de la LGTBfobia como parte constitutiva de la idiosincrasia del recién llegado (antaño, el andaluz; hoy, el africano) y del eterno extraño (el gitano) es otra más de las atribuciones negativas (como la delincuencia, la suciedad o el fundamentalismo) que constituyen al «exterior constitutivo» (Derrida, 1972) que amenaza a la modernidad, esa otredad que es fundamento y requisito para consolidación de la propia identidad. Sin pretender negar que entre las poblaciones señaladas existen, muy probablemente, personas con actitudes hostiles, e incluso violentas, hacia las expresiones LGTB, los discursos tendentes a enfatizar la LGTBfobia del Otro conllevan un doble peligro que es necesario neutralizar. Por un lado, tienen un efecto tranquilizante y desmovilizador para el Nosotros, percibido como una comunidad de pertenencia en la que la violencia y la discriminación sexogenérica son un problema menor o ya superado. Por otro lado, estos discursos de la otredad LGTBfóbica, aún formulados de forma ingenua, consolidan los fundamentos sobre los que se asientan los proyectos políticos y sociales de carácter racista y xenófobo tan fortalecidos en los últimos tiempos, y que cada vez más incluyen la tolerancia hacia lo LGTB entre los rasgos que vendrían a demostrar la — supuesta - superioridad cultural y moral del blanco occidental.

Como vemos, determinados barrios periféricos son señalados por algunas de las personas LGTB entrevistadas como territorios hostiles, al estar «dominados» por inmigrantes pertenecientes a culturas intolerantes y amenazantes para la diversidad sexual y de género. A esta intolerancia originaria se le añade a menudo un marcador económico-social como la pobreza. De este modo, el origen cultural, la identidad racializada y la clase social se convierten en las 
variables explicativas de la intolerancia periférica: «En los barrios periféricos hay muchos inmigrantes y personas pobres, gente con un nivel cultural más bajo, y es ahí donde puede haber más problemas» (hombre gay, 61 años. Sabadell). En el otro lado del espectro, el centro de la ciudad de Girona es percibido como una zona confortable "porque es donde hay mucho turista" (Mujer lesbiana, 58 años). Así pues, mientras que el binomio inmigrantelpobre funciona como una representación asociada a los principales peligros que acechan las ciudades actuales (y que a menudo son articulados recurriendo al poder normativo de conceptos como la convivencia, la cohesión o el civismo), la dupla turista/rico representa la vanguardia civilizatoria del pleno respeto a la diversidad.

En todo caso, el simple supuesto de que los barrios periféricos son un territorio hostil para las expresiones LGTB conlleva los riesgos de convertirse en una "profecía autocumplida». De acuerdo con Merton (1949), la profecía que se autorrealiza es aquella definición falsa de una situación que genera una serie de comportamientos y actitudes que acaban por convertir en verdadera esa falsa concepción original. En nuestro caso, la afirmación de que la periferia es intolerante o LGTBfóbica impide que se celebren manifestaciones de la diversidad sexogenérica en estas zonas, lo que acaba por dificultar que sus habitantes se familiaricen con dicha diversidad:

Con la compañía de teatro con la que estoy ahora hacemos un espectáculo [sobre diversidad sexogenérica] en una plaza del centro, al lado del Ayuntamiento. Cuando les planteo a mis compañeras de hacer la performance en Can Gambús, un barrio fuera del centro, me dicen que no saben si será lo mismo. Y si les digo de hacerlo en Can Puiggener [barrio periférico identificado como hostil], me dicen: «iAllí nos lincharán!». (Hombre gay, 38 años. Sabadell)

En un sentido similar, uno de los participantes en la investigación de Girona afirma:

Algunas parejas que conozco viven en Salt [pueblo colindante a Girona con un elevado porcentaje de población migrante], y no tienen ningún problema. Pero sí que..., ahora te hablo desde el desconocimiento, pero sí que me imagino que, si te morreas con otro tío en el medio de la calle Mayor de Salt, un «maricón» te lo escucharás seguro. Ahora he quedado muy bien con el colectivo de inmigrantes [rie]. (Hombre gay, 22 años)

Esta cita refleja que el participante es consciente de la carga racista de su mensaje, así como de la reproducción de estereotipos y del desconocimiento del territorio del que habla. Con todo, se ha de tener en cuenta que en una investigación como la que se ha llevado a cabo para la elaboración del presente artículo, el entrevistador representa a una institución académica $-\mathrm{y}$ a un determinado proyecto de investigación-, lo que condiciona el diálogo e influye en la producción del discurso de la persona entrevistada. De este modo, el entrevistado opta por justificar su posicionamiento tras verbalizar el prejuicio. Mantiene un argumento de «verdad» al asociar la migración con una 
menor tolerancia a la diversidad sexogenérica, a pesar de que conoce parejas del mismo sexo que viven en Salt y que no tienen ningún problema al respecto. El estereotipo racista prevalece así sobre las experiencias conocidas.

Otro participante se refiere a Salt con la frase de «no quiero ni pensar lo que debe ser en Salt» (hombre gay, 40 años), refiriéndose a la vida de las personas LGTB que residen en dicho municipio. Los territorios a los que se les atribuye una mayor carga migrante y en los que residen las clases populares y obreras son representados como espacios en los que la diversidad sexogenérica es algo impensable, indeseable o invivible. Sin embargo, cuando esta representación se contrasta con las experiencias de las personas LGTB que residen en estos territorios señalados, los «riesgos» asociados a las clases extranjeras y populares son, en muchos de los casos, estereotipos que reafirman las jerarquías sociales. A este respecto, algunas de las personas entrevistadas de Sabadell y Girona rechazan la tesis de la socioculturalización de la LGTBfobia, y destacan algunas potencialidades de los barrios periféricos en cuanto a la aceptación de la diversidad:

Se presupone que la zona del centro de las ciudades es la más tolerante con todo tipo de personas, por lo que la gente va al centro pensándose que tiene una serie de derechos que en las barriadas no existen [...] Pero muchas veces nos encontramos con la sorpresa de que en las barriadas se acepta mejor la diversidad porque están formadas por elementos y componentes de diversidad. (Profesional del sector educativo. Sabadell)

En todo caso, es importante destacar que, en muchas ocasiones, las percepciones sobre la LGTBfobia no tienen que ver tan solo con el entorno, sino también con los recursos personales y sociales que ayudan a hacer frente a las adversidades por tener una orientación sexual y/o una identidad de género no hegemónica. El capital cultural, las redes afectivas, el origen sociocultural, los recursos económicos, la edad o el género juegan un papel crucial en las experiencias y las percepciones del colectivo LGTB. De este modo, las personas con mayor capital cultural, económico y social tienen más estrategias y alternativas para hacer frente a las situaciones de discriminación. A modo de ejemplo, un hombre gay de mediana edad, propietario de una empresa y perteneciente a una familia burguesa de Sabadell «de toda la vida» consideraba que no había experimentado ningún tipo de discriminación, porque, en sus propias palabras, «somos [él y su esposo] una referencia en la ciudad». El origen sociocultural y el éxito profesional parecen conceder a esta persona un elevado estatus, que aparentemente no se ve afectado por su orientación sexual (socialmente más aceptable en tanto que está casado con su novio de siempre). Por tanto, las posiciones vitales están atravesadas por múltiples factores de privilegio y desigualdad que dotan a cada persona de distintos niveles de recursos y de poder para hacer frente a las adversidades. Por todo ello, pensar que la liberación de las personas LGTB depende únicamente de la cuestión identitaria es una falacia que conlleva ignorar otras lógicas sociales que determinan distintas posiciones, capacidades y posibilidades. 


\section{Conclusiones: pensando desde fuera de la gran ciudad}

Los estudios sobre diversidad sexual y de género en el entorno urbano todavía son muy embrionarios en España. Por tanto, nos encontramos ante la oportunidad de superar las lógicas de la gran ciudad en la configuración de este nuevo campo de estudio en los contextos de proximidad. Si somos capaces de subvertir las lógicas de la gran ciudad como espacio hegemónico para la producción de significados, discursos y representaciones de la diversidad sexual y de género contaremos con una aproximación más rigurosa y compleja de esta temática. Pero, sobre todo, contribuiremos a construir una imagen de la diversidad sexogenérica más inclusiva respecto a otras experiencias que no pasan por la gran ciudad. Los estudios anglosajones tardaron casi dos décadas en ofrecer miradas alejadas de la gran ciudad, reificando una y otra vez la gran urbe como espacio propio de «lo LGTB».

Habida cuenta de que las personas LGTB no solo viven en las grandes ciudades, es necesario darles a todas ellas espacios de visibilidad en las investigaciones sociales para contar con estudios que puedan reflejar la realidad más amplia y compleja. En el caso catalán, si centramos el foco únicamente en Barcelona, reducimos la mirada a un escenario que solo representa una parte de la población LGTB de Cataluña. Por el contrario, si somos capaces de significar lo LGTB más allá de los grandes eventos, los escenarios de socialización y los espacios dominantes de visibilización concentrados en Barcelona, podremos ofrecer nuevas miradas y análisis sobre las geografías de la diversidad sexogenérica.

No obstante, la gran ciudad no puede ser del todo ignorada. En los casos de Girona y Sabadell se demuestra que la influencia de Barcelona es fundamental en la constitución de las historias LGTB locales. Las idas y venidas a Barcelona son innumerables, significativas y están siempre presentes en las conversaciones de las personas LGTB de ambas ciudades. Aun así, hay que matizar que esta influencia no es única ni unidireccional. Por un lado, Barcelona sedimenta los comportamientos y las experiencias de sexiliados y visitantes puntuales. Por otro lado, las dinámicas globales que proyectan representaciones y costumbres ejercen también una influencia importante en ambas ciudades sin pasar por el filtro previo de Barcelona. Es decir, la cultura LGTB globalizada se amplifica, además, a través de Internet, de los viajes de ocio o de trabajo, así como a través de otras experiencias que las personas LGTB adquieren al margen de la gran ciudad. En resumen, son las interacciones constantes entre la gran ciudad de referencia (Barcelona), las dinámicas globales y las particularidades locales las que configuran las experiencias, las representaciones y los discursos LGTB de Girona y Sabadell. Todo ello teniendo en cuenta las trayectorias vitales, los recursos y las posiciones de poder de cada persona, así como el contexto social y cultural del lugar donde habita.

Finalmente, se ha visto que la construcción de alteridades culturales y económicas resulta determinante en las percepciones espaciales de la LGTBfobia. La vinculación de la LGTBfobia con una otredad configurada por marcadores 
étnico-clasicistas contribuye a la ordenación simbólica del espacio urbano a través de la construcción social de la dicotomía seguridad/inseguridad. Al mismo tiempo que advertir de las peligrosas consecuencias de estas representaciones, se ha subrayado la importancia de factores tales como el género, la edad, las redes afectivas o la capacidad económica a la hora de percibir y gestionar las situaciones de violencia y discriminación.

\section{Referencias bibliográficas}

AdLer, Sy y Brenner, Johanna (1992). «Gender and space: Lesbians and gay men in the city». International Journal of Urban and Regional Research, 16 (1), 24-34. <https://doi.org/10.1111/j.1468-2427.1992.tb00463.x>

Aldrich, Robert (2004). «Homosexuality and the City: An Historical Overview». Urban Studies, 41 (9), 1719-1737. <https://doi.org/10.1080/0042098042000243129>

ANNES, Alexis y REDLIN, Meredith (2012). «Coming out and Coming Back: Rural Gay Migration and the City». Journal of Rural Studies, 28 (1), 56-68. $<$ https://doi.org/10.1016/j.jrurstud.2011.08.005>

BaILEY, Robert (1999). Gay Politics, Urban Polities: Identity and Economics in the Urban setting. Nueva York: Columbia University Press.

BeCH, Henning (1997). When Men Meet: Homosexuality and Modernity. Chicago: The University of Chicago Press.

BeLL, David (2000). «Farm Boys and Wild Men: Rurality, Masculinity, and Homosexuality». Rural Sociology, 65 (4), 547-561. <https://doi.org/10.1111/j.1549-0831.2000.tb00043.x>

Bell, David y Valentine, Gill (eds.) (1995). Mapping Desire: Geographies of Sexuality. Nueva York: Routledge.

Black, Dan; Gates, Gary; Sanders, Seth y Taylor, Lowell (2002). "Why do gay men live in San Francisco?». Journal of Urban Economics, 51 (1), 54-76. <https://doi.org/10.1006/juec.2001.2237>

BOELlSTORFF, Tom y LeAP, William (2004). «Introduction: Globalization and "New" Articulations of Same-Sex Desire». En: LeAp, William y BOELlSTORFF, Tom (eds.). Speaking in Queer Tongues: Globalization and Gay Language. Urbana: University of Illinois Press, 1-21.

BROWN, Gavin (2008). «Urban (Homo)Sexualities: Ordinary Cities and Ordinary Sexualities». Geography Compass, 2 (4), 1215-1231. $<$ https://doi.org/10.1111/j.1749-8198.2008.00127.x>

- (2012). «Homonormativity: A Metropolitan Concept That Denigrates "Ordinary" Gay Lives». Journal of Homosexuality, 59 (7), 1065-1072. $<$ https://doi.org/10.1080/00918369.2012.699851>

BROWNE, Kath (2011). «Beyond Rural Idylls: Imperfect Lesbian Utopias at Michigan Womyn’s Music Festival». Journal of Rural Studies, 27 (1), 13-23. $<$ https://doi.org/10.1016/j.jrurstud.2010.08.001>

CÁCeres Feria, Rafael y VAlCUende del Río, José María (2014). «Globalización y diversidad sexual, gays y mariquitas en Andalucía». Gazeta de Antropología, 30 (3).

CASTELlS, Manuel (1983). The City and the Grassroots. Berkeley: University of California Press.

Champion, Tony y Hugo, Graeme (eds.) (2016). New Forms of Urbanization: Beyond the Urban-Rural Dichotomy. Londres y Nueva York: Routledge. 
Chauncey, G. (1994). Gay New York: Gender, urban Culture, and the making of the gay male world 1890-1940. Nueva York. Basic Books.

Coll-Planas, Gerard (dir.) (2017). Osona. Una comarca amb tots els colors: Pla marc per la igualtat per raó d'orientació sexual i identitat de gènere a Osona. Recuperado el 11 de abril de 2019, de <https:/www.vic.cat/viure-a-vic/dones/documentsdinteres/pla-marc-per-la-igualtat-per-rao-dorientacio-sexual-i-identitat-de-generea-osona/at_download/file>.

Delgado, Manuel (2011). El espacio público como ideología. Madrid: Catarata.

Derrida, Jacques (1972). Positions. París: Les Éditions de Minuit.

Duggan, Lisa (2002). "The New Homonormativity: The Sexual Politics of Neoliberalism». En: Castronovo, Russ y Nelson, Dana D. (eds.). Materializing Democracy. Durham y Londres: Duke University Press, 175-194.

ERIBON, Didier (1999). Réflexions sur la question gay. París: Flammarion.

GuZMÁN, M. (1997). “Pa la escuelita con mucho cuida’o y por la orillita”: A Journey through the Contested Terrains of the National and Sexual Orientation». En: Negro-Muntaner, Frances y Grosfoguel, Ramón (eds.). Puertorican Jam: Essays on Culture and Politics. Mineapolis: University of Minnesota Press, 209-228.

Halberstam, Judith (2005). In a Queer Time and Place: Transgender Bodies, Subcultural Lives. Nueva York: New York University Press.

HibBins, Ray (2005). «Migration and Gender Identity among Chinese Skilled Male Migrants to Australia». Geoforum, 36 (2), 167-180. <https://doi.org/10.1016/j.geoforum.2003.10.003>

Higgs, David (1999). Queer Sites: Gay Urban Histories Since 1600. Londres y Nueva York: Routledge.

Hindle, Paul (1994). "Gay Communities and Gay Space in the City». En: WhitTle, Stephen (ed.). The Margins of the City: Gay Men's Urban Lives. Aldershot: Arena, 7-25.

HuARD, Geoffroy (2014). Los antisociales: Historia de la homosexualidad en Barcelona y Paris, 1945-1975. Madrid: Marcial Pons.

HubBard, Philip (1999). Sex and the City: Geographies of Prostitution in the Urban West. Aldershot: Ashgate.

HubBard, Philip y WiLKInsOn, Eleanor (2015). «Welcoming the World?: Hospitality, homonationalism, and the London 2012 Olympics». Antipode, 47 (3), 598-615. <https://doi.org/10.1111/anti.12082>

Ingram, Gordon Brent; Bouthillette, Anne-Marie y ReTter, Yolanda (eds.) (1997). Queers in space: Communities, public places, sites of resistance. Seattle: Bay Press.

KAZYAK, Emily (2011). «Disrupting Cultural Selves: Constructing Gay and Lesbian Identities in Rural Locales». Qualitative Sociology, 34 (4), 561-581. <https://doi.org/10.1007/s11133-011-9205-1>

KnOPP, Lawrence (1992). "Sexuality and the Spatial Dynamics of Capitalism». Environment and Planning D: Society and Space, 10, 671-689. $<$ https://doi.org/10.1068/d100651>

- (1994). «Social Justice, Sexuality and the City». Urban Geography, 15 (7), 644-660. <https://doi.org/10.2747/0272-3638.15.7.644>

- (1998). "Sexuality and Urban Space: Gay Male Identity Politics in the United States, the United Kingdom, and Australia». En: FInCHER, Ruth y JACOBS, Jane N. (eds.). Cities of Difference. Nueva York: Guilford Press, 149-176.

KUHAR, Roman y ŠvAB, Alenka (2014). «The Only Gay in the Village?: Everyday Life of Gays and Lesbians in Rural Slovenia». Journal of Homosexuality, 61 (8), 1091-1116. <https://doi.org/10.1080/00918369.2014.872492> 
La FounTain-STOKES, Lawrence (2004). «De Sexilio(s) y Diáspora(s) Homosexual(es) Latina(s): Cultura Puertorriqueña y Lo Nuyorican Queer». Debate Feminista, 29, 138-57.

Langarita Adiego, Jose Antonio y Salguero VelázQuez, Maria Alejandra (2016). «Sexiled in Mexico City: Urban Migrations Motivated by Sexual Orientation». Bulletin of Latin American Research, 36 (1), 68-82. <https://doi.org/10.1111/blar.12523>

LoyD, Bonnie y ROWNTREe, Lester (1978). «Radical feminists and gay men in San Francisco: Social place in dispersed communities». En: LANEGRAN, David A. y Palm, Risa (eds.). An Invitation to Geography. Nueva York: McGraw Hill, 78-88.

MartíneZ-SAn Miguel, Yolanda (2011). "Sexilios: Hacia una nueva poética de la erótica caribeña». América Latina Hoy, 58, 15-30.

Mas Grau, Jordi y JubanY, Olga (2019). «De la révolution sexuelle à l'inclusion sociale des expressions LGBT+. Le processus d'obtention des droits LGBT en Espagne». Droit et cultures, 77 (1), 53-68.

MCGLYNN, Nick (2018). "Slippery Geographies of the Urban and the Rural: Public Sector LGBT Equalities Work in the Shadow of the "Gay Capital"». Journal of Rural Studies, 57, 65-77. <https://doi.org/10.1016/j.jrurstud.2017.10.008>

MÉRIDA JimÉNEZ, Rafael (2016). Transbarcelonas: Cultura, género y sexualidad en la España del siglo XX. Barcelona: Bellaterra.

MERTON, Robert K. (1949). Social theory and social structure: Toward the codification of theory and research. Glencoe, Illinois: The Free Press.

MirA, Alberto (2004). De Sodoma a Chueca: Una historia cultural de la homosexualidad en España en el siglo XX. Barcelona y Madrid: Egales.

Mort, Frank y NeAD, Lynda (eds.) (1999). Sexual Geographies, New Formations 37. Londres: Lawrence and Wisthart Press.

Muller, Tiffany (2013). "Ordinary (small) cities and LGBQ lives». ACME: An International E-Journal for Critical Geographies, 12 (2), 279-304.

NASH, Catherine (2013). "The Age of the "Post-Mo"?: Toronto's Gay Village and a New Generation». Geoforum, 49, 243-252. <https://doi.org/10.1016/j.geoforum.2012.11.023>

Ortega Arjonilla, Esther y Platero Méndez, R. Lucas (2015). «Movimiento feminista y trans* en la encrucijada: Aprendizajes mutuos y conflictos productivos». Quaderns de Psicologia, 17 (3), 17-30. $<$ https://doi.org/10.5565/rev/qpsicologia.1280>

Phillips, Richard; WATt, Diane y ShutTLETOn, David (eds.) (2000). De-Centring Sexualities: Politics and Representations beyond the Metropolis. Londres y Nueva York: Routledge.

Platero, Raquel (Lucas) (ed.) (2012). Intersecciones: Cuerpos y sexualidades en la encrucijada. Barcelona: Bellaterra.

PROBYN, Elspeth (1995). «Lesbians in space: Gender, sex and the structure of missing». Gender, Place and Culture, 2 (1), 77-84. <https://doi.org/10.1080/09663699550022107>

PUAR, Jasbir (2007). Terrorist assemblages: Homonationalism in queer times. Durham y Londres: Duke University Press.

RODÓ-DE-ZÁrATE, Maria (2015). «Young lesbians negociating public spaces: An intersectional approach through places». Children's Geographies, 13 (4), 413-434. <https://doi.org/10.1080/14733285.2013.848741> 
Sibalis, Michael (2004). "Urban Space and Homosexuality: The Example of the Marais, Paris' "Gay Ghetto"”. Urban Studies, 41 (9), 1739-1758. <https://doi.org/10.1080/0042098042000243138>

Smith, Geoffrey (2012). «Sexuality, Space and Migration: South Asian Gay Men in Australia». New Zealand Geographer, 68 (2), 92-100. <https://doi.org/10.1111/j.1745-7939.2012.01229.x.>

VasqueZ del Aguila, Ernesto (2014). Being a Man in a Transnational World: The Masculinity and Sexuality of Migration. Londres y Nueva York: Routledge.

Viveros VigoYa, Mara (2016). "La interseccionalidad: Una aproximación situada a la dominación». Debate Feminista, 52, 1-17.

Weston, Kath (1995). "Get thee to a big city: Sexual imaginary and the great gay migration». GLQ: A Journal of Lesbian and Gay Studies, 2, 253-277. <https://doi.org/10.1215/10642684-2-3-253>

WhitTle, Stephen (ed.) (1994). The Margins of the City: Gay Men's Urban Lives. Aldershot: Arena.

WiEnke, Chris y HiLl, Gretchen J. (2013). «Does Place of Residence Matter?: RuralUrban Differences and the Wellbeing of Gay Men and Lesbians». Journal of Homosexuality, 60 (9), 1256-1279.

<https://doi.org/10.1080/00918369.2013.806166> 